\title{
Caracterización de las Instituciones de Educación Superior (IES) en Chile: una aproximación a un modelo futuro de universidad (Principales reformas que se han presentado en el modelo de educación superior en Latinoamérica impactando en la mercantilización de las universidades)
}

\section{Characterization of Chilean Higher Education Institutions (HEI): an approach towards a future model of university (Main reforms applied in the Latin-American higher education model that made an impact in the commercialization of the universities)}

\author{
Rafael Jaime Carmona-López ${ }^{1}$ \\ Universidad Pontificia Bolivariana \\ Medellín, Colombia \\ rafaeljaimecarmona@hotmail.com \\ Iván Darío Toro-Jaramillo \\ Universidad de Medellín. \\ Medellín, Colombia \\ idtoro@udem.edu.co \\ José Antonio Riascos-Gonzales ${ }^{3}$ \\ Universidad Pontificia Bolivariana \\ Medellín, Colombia \\ josea.riascos@upb.edu.co
}

Recibido: 10 diciembre 2012 Aceptado: 26 junio 2014 Corregido: 27 junio 2014

Resumen: El contexto de la investigación que recoge este artículo busca mostrar a partir de la evolución y el desarrollo de las universidades y su direccionamiento en América Latina, y de modo particular en Chile, los

\footnotetext{
$1 \quad$ Doctor ( en ciencias de la administración universidad de Santiago de Chile. Actualmente laboro como profesor titular de la Universidad Pontificia Bolivariana en Colombia y profesor part time de la universidad Diego Portales en Chile. Ha participado como ponente en congresos nacionales e internacionales, entre sus publicaciones se destaca la pobreza como eje de la ética en un mundo globalizado, revista Ciencias estratégicas UPB. 2011. Dificultades para conformar el un clúster relacionado con el cuero y el calzado en el departamento de Antioquia, revista universidad cooperativa de Colombia, 2007. Competitividad y retos en la productividad del clúster textil-confección. Diseño y moda en Antioquia. Revista ciencias estratégicas 2008. Miembro del grupo de investigación, estudios empresariales, categoría B en Colciencias. Colombia y Investigador del grupo de investigación Gestión estratégica de instituciones de educación superior Chile.

2 Doctor en Filosofía y Doctor en Teología de la Universidad de Navarra, España. profesor jornada completa en la universidad de Medellin en Colombia. Con publicaciones tales como Libro. TORO JARAMILLO, I. D. y PARRA RAMÍREZ, R. D.: Método y conocimiento. Metodología de la investigación, Fondo Editorial EAFIT, Medellín 2006, 387 pp. ISBN 958-8281-11-3 Artículo: Clero insurgente y clero realista en la Revolución colombiana de la Independencia, en: Anuario de Historia de la Iglesia. Conmemoración del bicentenario de la Independencia de América Latina (1810-2010), XVII, Servicio de Publicaciones. correo electrónico: itorojar@gmail.com

3 Magister en Administración y especialista en Finanzas de la Universidad EAFIT de Medellín Colombia. Ingeniero Químico y especialista en negociación Internacional de la Universidad Pontificia Bolivariana (UPB). Actualmente profesor titular y consultor empresarial de la UPB. Ha participado como ponente en congresos de CLADEA en Chile y Colombia. Autor de artículos de revista como el direccionamiento estratégico de la Escuela de Ciencias estratégicas de la UPB. Miembro fundador del grupo de estudios Empresariales de la UPB Categorizado en Colciencias en B. Miembro de tanque de pensamiento del Plan Estrategico de Antioquia.PLANEA. Autor del libros: Servicio para la competitividad y La Gestión Estratégica Integral GESI. Miembro de observatorio estratégico de la UPB.
} 
distintos modelos de universidad, que llegan incluso a repercutir en el desarrollo de las sociedades, tanto pasadas como contemporáneas, y de los que es posible destacar algunas características similares y diferenciales que estos presentan. Cabe anotar que el modelo de educación chileno se ha considerado de gran impacto en algunos países de América Latina, especialmente en Colombia. Además, se planteó como objetivo general de esta investigación el reflexionar acerca de las reformas de la educación superior en América Latina, y el impacto que estas reformas han tenido en los últimos años en Chile, las cuales parecen haber generado una alta inconformidad en el contexto social, debido a la excesiva mercantilización de los servicios educativos. Para el desarrollo de este trabajo se realizó una revisión amplia de literatura que da cuenta del fenómeno a investigar y se realizaron entrevistas semi-estructuradas a expertos en el tema que ayudaron a comprender lo que los autores explican en la bibliografía en relación con los modelos y reformas de la educación en América Latina y la actual situación de la educación en Chile, consiguiendo de esta manera una clara visión sobre la realidad del problema.

Palabras claves: Modelos de universidades, reformas educativas en Chile, educación superior, prospectiva de la universidad, mercantilización de la universidad.

\begin{abstract}
The research context that this article seeks to show the different models of college, from the evolution and development of universities and their management in Latin America, particularly in Chile, and how they affect the development of societies, both past and contemporary, highlighting some similar and different characteristics that they have. It should be noted that the Chilean model of education has been considered to have a great impact on some countries in Latin America, especially in Colombia. Moreover, the general objective of this research was the reflection on the reforms of higher education in Latin America and the impact these reforms have had in recent years in Chile, which seem to have created a high dissatisfaction in the social context, due to the excessive commercialization of educational services. For the development of this paper an extensive review of the literature, which accounts for the phenomenon to be investigated is made and semi-structured interviews were carried out to experts in the field, which helped to understand what authors explain in the literature concerning models and educational reforms in Latin America and the current situation of education in Chile, thereby achieving a clear vision of the reality of the problem.
\end{abstract}

Keywords: Models of universities, educational reforms in Chile, higher education, prospective college commercialization of the university.

\title{
Introducción
}

De acuerdo con Brunner y Peña (2011) la primera universidad formal en la historia de occidente se remonta al año 1215, en Bolonia, donde aparece lo que podríamos considerar la estructura formal de una universidad, que coincide con la presentación del texto de Rhetorica Antiqua, del profesor Buoncompagno (c. 1165 - c. 1240). Casi al mismo tiempo, pero con características diferentes, surgió la Universidad de París (1231). Por su parte, Federico II Hohenstaufen, Rey de Sicilia y Jefe del Imperio Romano, es uno de los primeros soberanos en crear una universidad estatal en Nápoles (1224), hecha pública mediante un generalis lictera, reservándose el derecho de conferir títulos académicos. También los autores explican que en el año 1229 se inicia la primera universidad papal, fundada por Gregorio IX en Tolosa.

En esta perspectiva, explica el entrevistado $1^{4}$, que la palabra universidad al derivar del latín, Universitas, significa corporación o gremio y en este sentido hacía referencia al gremio de maestros

Entrevistado 1. Entrevista en Santiago de Chile, octubre de 2011 
y estudiantes. Por lo anterior, podemos pensar que las universidades surgen, en el siglo XIII, en un contexto imperial, donde sus propietarios eran los reyes, y en un contexto confesional, con un direccionamiento de la Iglesia católica.

Para el caso de Chile el inicio de la educación superior se da por la intervención del Estado, allí podemos anotar que la primera universidad fue la Universidad de Chile, fundada en el año 1842. Posteriormente se crea la Universidad Católica de Chile, en 1888 (después Pontificia), y luego, en el siglo XX, las Universidades de Concepción (1919), Católica de Valparaíso (1928), Universidad Técnica Federico Santa María (1931), Técnica del Estado (1947), Austral de Chile (1954) y, finalmente, la Universidad del Norte, posteriormente Universidad Católica del Norte, (1956). Estas ocho instituciones, dos estatales y seis privadas (tres vinculadas a la Iglesia Católica, otras tres de carácter no confesional), consolidaron el sistema de educación superior en Chile en aproximadamente un siglo (Brunner, 2009 a; Bernasconi y Rojas, 2004).

En esta perspectiva, en América Latina, la primera universidad que se creó fue la Universidad Nacional Mayor de San Marcos, fundada el 12 de mayo de 1551 en Perú.

Desde estos años hasta la actualidad se puede observar la evolución y el desarrollo de cada universidad y su direccionamiento, de acuerdo con su misión. Existe, además, una caracterización de la universidad de acuerdo con dos miradas: una desde la orientación investigativa, de tradición alemana, la Universidad de Berlín (1810), cuya formación estaba direccionada a la investigación, bajo el influjo de Alexander Von Humboldt y, la otra, la universidad napoleónica cuyo centro de formación era la transmisión de conocimientos, donde prima la información y los datos, contrario a la universidad investigativa en la que su principal eje es la producción de conocimiento (Toro y Parra, 2010).

Estas dos caracterizaciones de la universidad marcarían un camino en el desarrollo de la universidad por muchos años, hasta la actualidad. Hoy en día aún existe una alta incidencia de estos modelos, incluso en América Latina, llegando a repercutir en el desarrollo de las sociedades, tanto pasadas como contemporáneas. Aunque han sido varias las reformas a la educación superior emprendidas en América Latina, cabe destacar que en 1918 se realiza en Argentina, específicamente en la ciudad de Córdoba, una de las reformas más significativas que se han podido dar en la sociedad académica hasta nuestros días, y que a pesar de no haber sido del todo exitosa tuvo una gran transcendencia. Con ello se pedía que las universidades existieran como un organismo de los estudiosos para transmitir sus conocimientos a todo el pueblo y el laboratorio donde se analicen las ideas científicas, filosóficas, artísticas y sociológicas, con el propósito de dar una cultura en función social para una actuación consciente en las diversas manifestaciones del vivir individual y colectivo. De todas estas reformas, particularmente las que se han realizado en el siglo XX, pueden destacarse ciertos logros importantes pero también algunos desconciertos significativos, como es el caso de la alta mercantilización de la universidad, en algunos países, especialmente en Chile. Perdiéndose con ello la misión inicial de la universidad y la educación.

La tabla 1 hace una comparación de los diferentes modelos de universidades. Allí se presentan el modelo alemán, el modelo francés, el modelo de educación norteamericano y la tipificación de las universidades que pertenecen al Consejo de Rectores en Chile. 


\section{Tabla 1}

Comparativo entre modelos de universidades en diferentes contextos.

\begin{tabular}{|c|c|c|c|c|}
\hline $\begin{array}{l}\text { Modelos } \\
\text { Universitarios }\end{array}$ & $\begin{array}{l}\text { Modelo alemán } \\
\text { Universidad de } \\
\text { institutos }\end{array}$ & $\begin{array}{l}\text { Modelo francés } \\
\text { Universidades de la } \\
\text { academia }\end{array}$ & $\begin{array}{l}\text { Modelo norteamericano } \\
\text { Universidad } \\
\text { Departamento de } \\
\text { grado }\end{array}$ & $\begin{array}{l}\text { Universidades del } \\
\text { cruch } \\
\text { Consejo de rectores } \\
\text { de las Universidades } \\
\text { chilenas }\end{array}$ \\
\hline CONCEPCIÓN & $\begin{array}{l}\text {-Formar personas con } \\
\text { amplios conocimientos. } \\
\text { No necesariamente } \\
\text { relacionados con } \\
\text { las demandas de la } \\
\text { sociedad o del mercado } \\
\text { laboral. } \\
\text {--La investigación como } \\
\text { elemento estructurante } \\
\text { de las universidades. } \\
\text {-Taxativamente. } \\
\text { separada "ciencia" de } \\
\text { "profesión". }\end{array}$ & $\begin{array}{l}\text {-Formar a los } \\
\text { profesionales que } \\
\text { necesitaba el Estado. } \\
\text {-Centrada en la } \\
\text { docencia } \\
\text {-La universidad } \\
\text { al servicio de las } \\
\text { necesidades del } \\
\text { Estado. } \\
\text {-Profesionalizante. }\end{array}$ & $\begin{array}{l}\text {-Universidades surgen } \\
\text { como espacio donde se } \\
\text { genera investigación. } \\
\text {-Unidad de } \\
\text { investigación, docencia } \\
\text { y estudios. }\end{array}$ & $\begin{array}{l}\text { - Centrada en la } \\
\text { docencia. } \\
\text { - Llegar a ser una } \\
\text { universidad de } \\
\text { docencia con } \\
\text { investigación. }\end{array}$ \\
\hline $\begin{array}{l}\text { INSTITUCIONES } \\
\text { ENCARGADAS }\end{array}$ & $\begin{array}{l}\text {-La universidad } \\
\text { conformada por } \\
\text { institutos. } \\
\text {-Además de las } \\
\text { Universidades, } \\
\text { otros sectores no } \\
\text { universitarios } \\
\text { se involucran en } \\
\text { las actividades } \\
\text { investigativas. }\end{array}$ & $\begin{array}{l}\text {-La universidad } \\
\text { academia. } \\
\text {-Las écoles } \\
\text { pertenecían al sector } \\
\text { de la élite de la } \\
\text { educación superior } \\
\text { francesa. }\end{array}$ & $\begin{array}{l}\text {-Universidades con } \\
\text { sus departamentos de } \\
\text { grado y posgrados. }\end{array}$ & $\begin{array}{l}\text { Existen escuelas } \\
\text { que tienen a su } \\
\text { cargo diferentes } \\
\text { facultades. } \\
\text { Se pretende la } \\
\text { formación completa. }\end{array}$ \\
\hline FinANCIAMIENTO & $\begin{array}{l}\text {-Financiamiento } \\
\text { interno. } \\
\text {-Posteriormente } \\
\text { se suma el } \\
\text { financiamiento estatal } \\
\text { y el financiamiento del } \\
\text { sector privado. }\end{array}$ & $\begin{array}{l}\text {-Financiamiento pobre. } \\
\text {-Casi el } 90 \% \text { de sus } \\
\text { ingresos derivan del } \\
\text { tesoro nacional. } \\
\text {-No reciben } \\
\text { financiación de } \\
\text { ningún otro ente que } \\
\text { no sea el Estado. }\end{array}$ & $\begin{array}{l}\text {-Financiamiento } \\
\text { interno. } \\
\text {-Financiamiento } \\
\text { externo. } \\
\text {-Financiamiento } \\
\text { estatal. } \\
\text {-Financiación de la } \\
\text { educación superior en } \\
\text { los Estados Unidos } \\
\text { ha aumentado } \\
\text { considerablemente } \\
\text { en una comunidad } \\
\text { académica } \\
\text { extremadamente } \\
\text { competitiva. }\end{array}$ & $\begin{array}{l}\text {-Financiamiento } \\
\text { interno: } \\
\text { Recursos propios } \\
\text {-Financiamiento } \\
\text { estatal. }\end{array}$ \\
\hline
\end{tabular}

Nota: elaboración propia a partir de Oviedo Coronel, 2010 
Realizando una analogía con lo anterior se podría decir que los modelos de universidad desde sus inicios hasta la actualidad han tenido una evolución, a partir de ciertas variaciones de acuerdo con determinadas circunstancias. Independiente de la orientación que se tenga, lo anterior ha permitido que tanto el modelo napoleónico como el modelo de investigación o humboldtiano, conocido también como el modelo alemán, desde sus inicios hasta hoy presenten cambios, marcados bien por el desarrollo pragmático o por el desarrollo científico.

Este artículo se estructuró en relación con los siguientes temas: una caracterización de los modelos de educación en América Latina, a partir de la cual se quiere plantear una de las preocupaciones que mayor desconcierto ha ido generando en América Latina, como es lo relacionado con el mercado y mercantilización de las universidades en Chile, para luego, finalmente, hacer un aporte en relación con el análisis de los modelos de sistemas de educación superior. Lo anterior se consigue teniendo en cuenta el objetivo general de esta investigación: reflexionar acerca de las reformas de la educación superior en América Latina, y el impacto que estas reformas han tenido en los últimos años en Chile, las cuales parecen haber generado una alta inconformidad en el contexto social, debido a la excesiva mercantilización de los servicios educativos. Durante varios años se ha venido trabajando en esta investigación, con el apoyo de una amplia revisión de aquella literatura que da cuenta de la situación de la educación en el continente, para lo cual, además, se realizaron entrevistas semi-estructuradas a expertos que han jugado un papel importante dentro del sistema de educación en Chile, desde el Ministerio de Educación, como pares evaluadores, profesores de destacadas universidades, directores de centros de investigación, en Santiago de Chile, quienes ayudaron a comprender lo que los autores explican en la bibliografía en relación con los modelos y reformas de la educación en América Latina y la actual situación de la educación en Santiago de Chile, consiguiendo de esta manera una clara visión sobre la realidad del problema.

\section{Caracterización de los modelos de educación en América Latina}

Con respecto a la visión de Brunner, J. y Peña, C. (2011), cabe considerar que en la actualidad algunos países están incorporando modelos de experiencias distintos de los modelos mencionados, los cuales han tenido una alta estabilidad, tales como los modelos de educación superior de Suecia, Alemania, República de Corea, Dinamarca o Japón. En estos países es posible pensar las universidades en alguna de sus dimensiones como parte del aparato del Estado, llegando paulatinamente al olvido de los dos modelos tradicionales, el napoleónico y el alemán, permitiendo que las universidades adquieran, si así lo deciden, el estatus de entidad independiente, y reteniendo el Estado un gobierno a distancia mediante incentivos y políticas igualitarias hacia todo el sistema.

Para el entrevistado $2^{5}$, en el caso de América Latina, las universidades más representativas en los últimos años han sido aquellas con orientación hacia la investigación, entre las que destacan algunas universidades brasileñas y mexicanas, las cuales han sido pioneras en la investigación, con una orientación más científica, aunque también sobresalen otras universidades de la región

Entrevistado 2. Entrevista en Santiago de Chile, octubre de 2011. 
que han realizado grandes esfuerzos, faltándoles todavía un largo camino por recorrer hasta llegar a convertirse en universidades de investigación. En relación con lo anterior, también puede decirse que en América Latina existe una cierta crisis de identidad universitaria, y en muchos casos puede afirmarse que la universidad latinoamericana ha perdido el rumbo. Para este entrevistado 2, América Latina ha tenido como referente el modelo de Universidad de Norte América y, a su vez, las universidades norteamericanas han seguido el modelo alemán.

Centrándonos en los modelos educativos que ha buscado Chile, se ha tenido un desarrollo muy similar al de los otros países de América Latina: hay una expansión o crecimiento en el número de las matrículas, sin que exista un direccionamiento claro en relación con el tipo de universidad que se quiere, y más bien ha prevalecido el modelo de universidad profesionalizante, de sola docencia o transmisión de conocimientos. En el caso particular chileno se ha dado una mayor cobertura y ampliación de la matrícula con respecto al resto de países del continente.

En relación con el crecimiento en número de universidades, el entrevistado $3^{6}$ recuerda que las universidades en Chile para poder iniciar actividades debían involucrarse bajo alguna forma con participación y autorización del Estado, pues no se les permitía iniciar actividades de tipo académico sin la aceptación del Gobierno que en su momento ejerciera la autoridad.

Brunner (2009) argumenta que específicamente a partir de los años 50, se debaten las políticas necesarias para alinear los mercados de la enseñanza terciaria, donde destacan cuatro reformas representativas: la reforma universitaria del año 1967; la intervención de las universidades en el año de 1973, coetáneamente con la instauración de un gobierno militar; la implementación por ese Gobierno de una política de mercados para la educación terciaria, que comienza a implementarse a partir de $1981 \mathrm{y}$, por último, sus posteriores transformaciones a partir de 1990 con la recuperación de la democracia. La anterior afirmación coincide con la argumentación que hace Marshall (2010) donde indica que la educación terciaria ha transitado desde un sistema de estudios superiores elitista y poco diversificado, financiado principalmente por el Estado, hacia uno masivo y diversificado, que se financia mayoritariamente con recursos privados. Esto originó una notoria expansión del número de universidades -de 8 en 1980 a más de 60 en-, de estudiantes universitarios -de 130.000 en 1990 a casi 500.000- en el año 1990 y de profesionales titulados -de 13.000 en el mismo periodo a más de 35.000 egresados por año.

En la revisión de la literatura se pudo destacar cómo en América Latina se han dado reformas en la educación que han transcendido hasta los contextos actuales y han tenido una alta repercusión. Para el caso de Chile se subraya cómo la reforma del 90 posibilitó una alta masificación de la matrícula, con una característica bien determinada como fue la globalización, enmarcada en políticas de neoliberalismo, contribuyendo a reorganizar el sistema universitario. Sin embargo, y a pesar de lo anterior, según el entrevistado $4^{7}$, el sistema de educación que en la actualidad existe en Chile presenta unas variables de exclusión que no permiten que todas las personas puedan ingresar a la universidad, o si lo hacen presentan algunas restricciones de selectividad.

Lo anterior coincide con lo que afirman los distintos entrevistados, quienes explican que

Entrevistado 3. Entrevista en Santiago de Chile, octubre de 2011

Entrevistado 4. Entrevista en Santiago de Chile, octubre de 2011 
el aumento creciente de la privatización de la educación superior en Chile es un problema de fondo que tiene el país, descartando un problema ideológico, instrumental y económico. Con lo anterior se puede realizar una lectura donde se concluya que el colectivo de estudiantes reclama un modelo de educación de inclusión, donde existan unas políticas de educación que jueguen un papel importante. Las manifestaciones de los estudiantes le están recordando al país que aún existe un problema estructural, por lo que presionan al sistema de educación actual para encontrar una pronta solución, lo cual no se logra sin tener una fundamentación clara del problema y un modelo de educación que soporte el actual.

En esta misma línea el pensamiento, tanto Brunner (2009) entrevistado $3^{8}$ han planteado que la educación terciaria en Chile ha tenido un crecimiento marcado por la desigualdad y por la propiedad, teniendo en cuenta que a partir de la reforma de 1981 se dio un direccionamiento enmarcado en lo privado, por lo cual el país ha pasado de tener una educación superior subsidiada a tener una educación privada. Además, este entrevistado 3 argumenta que la inversión que realiza una persona en educación en relación con su tasa de recuperación presenta una desigualdad de acuerdo con su estrato socioeconómico y a las relaciones sociales que este tenga con el medio donde esté ubicado, teniendo en cuenta que la mercantilización de la educación superior no se ha dado de manera trasparente por parte de los actores involucrados, debido al alto desconocimiento que poseen las personas del común que toman la decisión de matricularse en un determinado programa y en una universidad especifica. La sociedad ha sido mal informada con un mercadeo basado en una publicidad en ocasiones engañosa, desconociendo la tasa de retorno de la inversión que se realiza en un programa de educación terciario, desconociéndose la calidad y lo más importante la demanda de los egresados que tiene el programa en el mercado laboral.

\section{Mercado y mercantilización de las universidades en chile}

En la visión de Clark (1998), el funcionamiento de este sistema debe ser coordinado (es decir, las reglas del mercado para la oferta y la demanda), para lo cual él lo representa en un triángulo, donde el vértice A representa al Estado, el vértice B representa a las Corporaciones y el vértice $\mathrm{C}$ representa al Mercado:

Históricamente, la coordinación de los sistemas de educación superior en el mundo giraron en torno a los vértices A/B (Estado - Corporaciones), en el caso europeo más centrado en A y en el caso latinoamericano más centrado en B. El sistema en Estados Unidos, desde el comienzo de su desarrollo, giró alrededor de C.

Sin embargo, en el último tiempo la mayoría de los sistemas de educación superior están acercándose al vértice C, o sea al del mercado (Clark, B. 1998) y la competencia entre instituciones en diversas formas. De acuerdo con este planteamiento, para el caso de Chile se podría afirmar que el colectivo de la educación superior se centra más hacia el vértice $\mathrm{C}$.

Efectivamente, resulta claro en la explicación que hace Donoso (2009) que existe una tendencia en general más hacia el mercado, a la vez que argumenta que la revisión del concepto

Entrevistado 3. Entrevista en Santiago de Chile, octubre de 2011 
escasez se puede dar como punto de partida para la comprensión del mercado, estando este asociado a la oferta y a la demanda. Desde la perspectiva de Ospina y Sanabria (2010), el mercadeo de servicios cobra importancia a partir de la década económicamente positiva de los año 1970, como consecuencia de la situación económica existente en ese momento en Norteamérica. Dicho periodo se caracterizó por la paulatina transformación de la economía: de una economía basada en la producción de bienes hacia una economía a partir de servicios; no obstante, para el caso de América Latina y especialmente de Chile su principal expansión en el mercado de servicios data del año 1981.

Es de aclarar que la reforma de 1981, en palabras de todos los entrevistados, hizo que se iniciara un proceso de mercantilización del sistema universitario en Chile, ingresando al mercado universitario empresarios que solo deseaban el lucro para los dueños de las universidades, olvidándose de la misión que debería tener la universidad en el contexto académico para repercutir en el contexto social, dándosele una gran importancia a programas que no necesitaban una alta inversión en espacios físicos, laboratorios y profesores de tiempo completo.

Es de anotar que Brunner (2010) destaca que en aquellas universidades en las que el comportamiento se define con fines de lucro no existe una mayor dificultad para aplicar el análisis económico estándar, pues se trata de firmas cuyo objetivo, a la manera de cualquier otra empresa comercial, es maximizar sus utilidades. Mas formalmente, esto significa que podemos esperar de estas instituciones que ellas eligen a su personal académico y demás insumos para producir un determinado nivel de output u oferta de cursos al más bajo costo posible, deteriorando la calidad e incrementando la cantidad de estudiantes. En ese orden de ideas esta afirmación no está lejos de las argumentaciones expresadas por la mayoría de los expertos entrevistados.

Ahora bien, cabe considerar que en relación con la argumentación que hace Brunner, Tillett, et al. (2005), no puede dejar de hacerse una crítica frente a los procesos de mercantilización de la educación, particularmente en América Latina y los países en vía de desarrollo.

En palabras del entrevistado $1^{9}$ una universidad que se caracterice por tener un pensamiento científico y que busque su complejidad debe tener facultades tales como: filosofía, sociología, teología. Lo cual lleva al estudiante y a su comunidad académica a reflexionar sobre diferentes tópicos, creándose con ello la interdisciplinariedad y la transdisciplinariedad del conocimiento.

Lo anterior nos lleva a reflexionar que aunque esta mercantilización de la educación superior en Chile no se ha declarado abiertamente, y que aún no han llegado multinacionales de la educación superior que cotizan en la bolsa comercial de Nueva York, como el caso de la Universidad de Phoenix, la más grande institución privada de educación post secundaria, sin embargo existen actores internos que tienen un monopolio del mercado. En síntesis, en la versión de los expertos entrevistados no solo la inversión que realiza el Estado para subsidiar la educación pública es muy poca, sino que ha direccionado todo su esfuerzo hacia la formación universitaria, desconociendo los programas técnicos. Si bien es cierto que la educación superior o terciaría no es solamente universitaria, también se debe considerar la formación técnica como factor de desarrollo en una economía.

Entrevistado 1. Entrevista en Santiago de Chile, octubre de 2011 
Fuhrman (2011) argumenta que en Norteamérica está ocurriendo lo siguiente: negar el acceso a créditos estatales o préstamos avalados por el Estado y restringir los créditos de bancos privados para aquellas instituciones de educación que no demuestren el cumplimiento de algunos indicadores básicos, como la tasa de retención de estudiantes, la tasa de graduación y la tasa de empleo efectivo en el campo profesional estudiado a cinco años de egresado, entre otros aspectos.

En la perspectiva de Fuhrman (2011) por desgracia hemos dejado al mercado decidir sobre la calidad de la educación. Una expresión que en muchos países en el mundo, y especialmente en Chile, se está pronunciando por estos días debido a las inconformidades de la sociedad.

Para tener un estado de referencia mucho más claro sobre la estructura de la educación superior en Chile, se destacan tres variables interesantes relacionadas con el sector de la educación, como son: la estructura del mercado, el comportamiento organizacional y el desempeño que ha tenido el sector de la educación superior en este contexto.

\section{Análisis de modelos de sistemas de educación superior}

Entre los modelos de instituciones de educación superior de mayor éxito en su desempeño, al reflejar altos estándares de calidad y una alta visibilidad en los rankings mundiales en la última década, analizaremos los siguientes modelos de universidades: Finlandia, Australia y Singapur, al considerar que han tenido una buena relevancia y gran visibilidad internacional, llegando a ocupar puestos significativos en algunas mediciones.

\section{Características del modelo de educación en Finlandia}

Como explica Halinen y Järvinen (2008) este sistema de educación es en un alto porcentaje público. El número de alumnos por grupo en la secundaria es reducido, se considera que es entre quince a veinte estudiantes por aula de clase, y existe una característica bien determinada como es la rigurosidad en el ingreso a la docencia, generándose con ello una alta demanda de aspirantes a ejercer dichos cargos. En Finlandia, los profesores son bien vistos en la sociedad, ganan un sueldo similar al del resto de los profesionales y son seleccionados de entre los mejores egresados de la educación media mediante un examen.

Es de aclarar que el Estado finlandés le transfiere un mayor grado de independencia a las universidades, establece procesos de estímulos, les brinda a las universidades una alta libertad para que inicien proyectos que signifique negocios; lo anterior lleva a que el Estado exija altos estándares generándose con ello una reciprocidad positiva.

\section{Modelo de Universidad en Australia}

Este modelo presenta unas características bien diferentes a nuestros modelos en América Latina. En palabras de James (2011), no todos los estudiantes que terminan la educación media o secundaria ingresan a la universidad. Cerca del $25 \%$ de los estudiantes son alumnos de otras partes del mundo que pagan por su educación (naciones de China, India y Asia sudoriental). Estos ingresos son muy importantes para el bienestar financiero del sector de la educación superior. 
El sistema de educación australiano se considera de gran calidad; allí destacan universidades con altos estándares y muestran muy buenos resultados en diversos planes de evaluación internacionales. En el Times Higher Education, en el 2011, las universidades australianas presentaron las siguientes posiciones: Melbourne $=37$, Universidad Nacional Australiana $=38$. En el Shanghai Jiao Tong: Universidad de Melbourne $=60$, Universidad Nacional Australiana $=70$. Lo anterior da cuenta del reconocimiento mundial que tienen estas universidades. Ocho universidades australianas destacan entre los 100 primeros lugares, 23 en los 500 primeros lugares. En dicho ranking aparece la Universidad de Chile entre las 500, en el puesto 401.

James (2011) explica que Australia cuenta con cuatro tipos de universidades, en términos generales: 1. Las dedicadas a la investigación (el Group of Eight), 2. Las universidades de 'tecnología' (la Australian Technology Network), 3. Las universidades 'metropolitanas' y 4. Las universidades rurales/regionales. Es relevante destacar la forma como en este sistema se seleccionan a los estudiantes: para que un estudiante pueda ingresar a la universidad debe hacerlo mediante la medición del ATAR (AustralianTertiaryAdmissions Rank), que es la principal herramienta de selección por parte de las universidades. Existe una gran presión de los núcleos familiares para que los estudiantes logren altos puntajes en ATAR, y así puedan ingresar a la universidad. Las escuelas privadas de elite tienen gran éxito al momento de apoyar a sus estudiantes.

Cabe considerar que los alumnos australianos presentan poca movilidad académica, generalmente donde terminan el ciclo de formación secundaria, allí inician su formación profesional. La mayoría de los estudiantes se quedan en el estado donde nacieron y viven en sus casas, contrario al fenómeno que se ve en Chile donde una gran mayoría de estudiantes provienen de las diferentes regiones y van a estudiar a las universidades de la capital.

En la descripción que realiza James (2011) la gran mayoría de los estudiantes australianos mantienen trabajos de media jornada (70\% trabaja, de los cuales la media es de 15 horas a la semana). Esto hace que se genere una alta preocupación entre los directivos de las instituciones, al considerar que es baja la dedicación al estudio, lo que podría ser una de las causas de la deserción en el primer año.

Los postulantes tienen algunas preferencias de cursos, cuyo atractivo aumenta según la selectividad de ingreso. Las evaluaciones de ingreso sirven como una variable del prestigio y la calidad.

\section{Modelo de universidad en Singapur}

La lengua utilizada para la enseñanza en Singapur es el inglés. Toda la ciudadanía debe a asistir a escuelas estatales hasta el sexto nivel de la educación primaria, con 12 años de edad. La población de niños y niñas asiste a los jardines de infancia previamente al año en que dan inicio con la educación primaria. Cada gobernante es el principal proveedor de la educación infantil a través de la división comunitaria (Blancas et al., 2013).

Si bien el inglés es la lengua madre en la enseñanza de las matemáticas y ciencias naturales, cada alumno perteneciente a la comunidad china puede asistir a la Special Assistance Plan (Plan de Ayuda Especial) en la cual reciben recursos adicionales para recibir enseñanza en chino como en inglés.

Al igual que en Chile, es el Estado por medio del Ministerio de Educación quien establece los niveles académicos, situación que colabora en eliminar una diferenciación entre 
las escuelas privadas y estatales, ya sea en recursos recibidos desde el gobierno central como en la autonomía de las políticas establecidas en el ingreso de los alumnos.

Es importante señalar, que existen ciertos centros educativos universitarios autónomos, estos son: la National University of Singapore, la Nanyang Technological University y la Singapore Management University, a las cuales se podría sumar un cuarto establecimiento denominado Singapore University of Design and Technology que aún se encuentra en el proceso de evaluación.

Para Morín "es necesario introducir y desarrollar en la educación el estudio de las características cerebrales, mentales y culturales del conocimiento humano, de sus procesos y modalidades, de las disposiciones tanto psíquicas como culturales que permiten arriesgar el error o la ilusión" (1999, p. 141). En el contexto de Chile resulta poco asertivo desarrollar las tres dimensiones, ya que se carece de una estructura clara que le permita al estudiante y a su núcleo familiar tener una concentración con base en lo planteado.

\section{Conclusión}

Cuando se piensa en la universidad es necesario no perder de vista cuál es la misión y compromiso con la sociedad. Desde sus orígenes, la universidad ha tenido una tarea bien definida, que en la mayoría de los casos se ha desarrollado y evolucionado a partir de los distintos modelos de universidad. La literatura consultada y las entrevistas con expertos muestran cómo Chile ha tenido un modelo de desarrollo muy parecido al de los otros países de América Latina, con un crecimiento en la matrícula, sin que exista un direccionamiento claro en relación con el tipo de universidad que se quiere. Asimismo, han sido de mucho impacto social las distintas reformas de la educación que han transcendido hasta los contextos actuales y han tenido una alta repercusión. En todo este contexto de discusiones debe preocuparnos hacia dónde se van dirigiendo las universidades. El acudir a otros referentes de universidades, a otros modelos de universidades, puede convertirse en un aliciente y acicate sin que se pierda de vista lo principal: lo que debe ser la misión de la universidad.

En síntesis, de todas estas reformas que se han buscado para la educación superior en América Latina, como se mencionó en el texto, pueden mencionarse algunos aciertos, principalmente en relación con la ampliación de la matrícula, que han servido para potenciar la universidad, pero también se tienen serios problemas que han hecho perder el rumbo a la universidad, debido en buena parte a la alta mercantilización de la universidad: se venden productos académicos y se ofertan portafolios de servicios educativos, perdiéndose con ello la misión fundamental de la universidad y la educación: la formación.

De igual modo, no solo es posible identificar con facilidad los diferentes actores del sistema educativo, tales como: el Estado, los profesores, los estudiantes, los empresarios y la sociedad en general, sino también reflexionar acerca de las distintas formas como cada uno de ellos está abordando el problema de la educación superior en el país.

En relación con el futuro de las universidades se considera importante, aunque no definitivo, que se pueda continuar observando la medición que se hace desde los diferentes rankings de las principales universidades en el mundo, tales como la medición de Shanghai Jiao Tong o el Times Higher Education, donde se miden aspectos como: el tamaño de la 
universidad por su nivel de investigación y los niveles de producción científica, aunque no pueden considerarse como el único referente de la universidad del futuro.

Sin bien los diferentes rankings pueden ayudar para que la universidad pueda verse en el contexto de las demás universidades, y potenciar así el trabajo académico e investigativo, sin embargo no pueden convertirse en la razón de ser las universidades, pues debe recordarse que cada uno de esos rankings lo que buscan es beneficiar a quienes los producen y responder a condiciones sociales, políticas, económicas y culturales muy diferentes a las nuestras en América Latina.

\section{Referencias}

Argandoña, G. (octubre, 2011) Entrevista con Guillermo Argandoña, Profesor de la faculta de ingeniería de la universidad de Santiago de Chile, Perspectiva de la educación superior en América Latina. [Grabación en audio].

Berlasconi, A. y Rojas, F. (2004). Informe sobre la Educación Superior en Chile: 1980-2003. Recuperado de http://unesdoc.unesco.org/images/0014/001403/140395s.pdf

Bernasconi, A. (2006). La difícil tarea de clasificar universidades. Calidad en la Educación. Santiago, Chile. Recuperado de: http://dialnet.unirioja.es/servlet/articulo?codigo $=2286748$

Bernasconi, A. (septiembre, 2011). Entrevista con Andrés Bernasconi, vicerrector académico de la Universidad Andrés Bello de Chile, Perspectiva de la educación superior en América Latina. [Grabación en audio].

Blancas, P., Caycho, C., Hinostroza, O., Ortíz, E., Rojas, J. y Rodríguez, Y. (2013). Singapur. Universidad Peruana Los Andes, Hunacayo, Perú, Recuperado de: https://www. academia.edu/5330276/SINGAPUR PAIS

Brunner, J., Tillet, A., Elacqua, G., Bonnefoy, J., González, S., Pacheco, P., Salazar, F. (2005). Guiar el Mercado. Informe sobre la Educación Superior en Chile. Viña del Mar, Chile.

Brunner, J. (2007). Educación superior en Chile: Instituciones mercados y políticas gubernamentales 1967-2007. Ediciones Diego Portales, Santiago, Chile.

Brunner, J. (2009). Políticas de Educación Superior: Tendencias internacionales. Recuperado de www.brunner.cl

Brunner, J. (2010). "El lugar del mercado en el presente y futuro de la educación superior chilena". En F. López Segrera y D. M. Rivarola (Comps.) La Universidad ante los desafíos del siglo XXI. Revista Paraguaya de Sociología. Quinquenio del Cincuentenario de la Revista, (pp. 765-836). 
Brunner, J. y Peña, C. (2011). El conflicto de las Universidades: entre lo público y lo privado. Ediciones Diego Portales, Santiago, Chile.

Brunner, J.J. (mayo, 2011). Entrevista con José Joaquín Brunner, ex ministro de Estado de Chile, Perspectivas de la educación superior en América Latina. [Grabación en audio].

Caicedo Escudero, J. (octubre, 2011). Entrevista con Jaime Caicedo Escudero, profesor del doctorado de la Facultad de Administración y Economía - FAE. Perspectiva de la educación superior en América Latina. [Grabación en audio].

Clark, B. (1998). La creación de universidades emprendedoras: Caminos de la organización y de la transformación. Oxford y Nueva York: Brunner (05 de mayo de 2011).

Donoso, S. y Schmal S. (2009). Introducción a la Economía de la Educación: El fenómeno educativo y su connotación económica. Talca, Chile: Editorial Universidad de Talca.

Donoso, S. (octubre, 2011). Entrevista con Sebastián Donoso, Director del Programa de Maestría en Política y Gestión, Universidad de Talca, Chile, Perspectiva de la educación superior en América Latina. [Grabación en audio].

Fuhrman, S. (2011, 25 de noviembre). Susan Fuhrman, experta de la U. de Columbia, participa en encuentro sobre calidad y desafíos de la educación [Facebook] Recuperado de: https://es-la.facebook.com/pages/Colegio-de-Profesores-RegionalValpara\%C3\%ADso/240585409334696

Halinen, I y Järvinen, R. (marzo, 2008). En pos de la educación inclusiva: el caso de Finlandia. Revista Perspectivas, 145(1), 97-127, París: UNESCO. Recuperado de http://www.ibe. unesco.org/fileadminin/user upload/Publications/Prospects/Prospects145 spa.pdf

James, R. (2011). Factores que influyen en las decisiones de los futuros estudiantes de educación superior. XVI Seminario internacional titulado: Equidad en el acceso a la educación superior del Consejo Nacional de Educación. Santiago, Chile.

Mahncke, M. (noviembre, 2011). Entrevista con Margarita Mahncke, profesora de la Universidad Diego Portales, Perspectiva de la educación superior en América Latina. [Grabación en audio].

Marshall, J. (2010). Educación Superior: Institucionalidad para los nuevos desafíos. Calidad de la educación, 236-251. 
Morín, E. (1999). Los siete saberes necesarios para la educación del fututo. Documento de la Unesco: Paris.

Ospina, M., y Sanabria, P. (2010). Un enfoque de mercadeo de servicios educativos para la gestión de las organizaciones de educación superior en Colombia: el modelo MIGME. Revista de la Facultad de Ciencias Económicas Universidad Militar Nueva Granada, 18(2), 107-136. Recuperado de http://www.scielo.org.co/pdf/rfce/v18n2/v1 8n2a07.pdf

Richards, H (octubre, 2011). Entrevista con Howard Richards, profesor del doctorado de la Facultad de Administración y Economía - FAE. Perspectiva de la educación superior en América Latina. [Grabación en audio].

Schiefelbein E. (agosto, 2011). Entrevista con Ernesto Schiefelbein, ex ministro de educación de Chile, Perspectiva de la educación superior en América latina. [Grabación en audio].

Toro, I. y Parra, R. (2010). Fundamentos epistemológicos de la investigación y la metodología de la investigación. Medellín: Fondo Editorial Universidad Eafit. 